\title{
Variations on the Petersen Colouring Conjecture*
}

\author{
François Pirot \\ Équipe Orpailleur, LORIA (Université de Lorraine, C.N.R.S., INRIA) \\ Vandouvre-lès-Nancy, France and \\ Department of Mathematics, Radboud University Nijmegen, Netherlands \\ francois.pirot@loria.fr \\ Jean-Sébastien Sereni \\ Centre National de la Recherche Scientifique (ICube, CSTB) \\ Strasbourg, France \\ sereni@kam.mff.cuni.cz \\ Riste Škrekovski \\ Faculty of Information Studies, Novo Mesto \\ Faculty of Mathematics and Physics, University of Ljubljana, and \\ FAMNIT, University of Primorska, Koper, Slovenia \\ skrekovski@gmail.com
}

Submitted: Feb 15, 2019; Accepted: Dec 9, 2019; Published: Jan 10, 2020

(C) The authors. Released under the CC BY-ND license (International 4.0).

\begin{abstract}
The Petersen colouring conjecture states that every bridgeless cubic graph admits an edge-colouring with 5 colours such that for every edge $e$, the set of colours assigned to the edges adjacent to $e$ has cardinality either 2 or 4 , but not 3 . We prove that every bridgeless cubic graph $G$ admits an edge-colouring with 4 colours such that at most $\frac{8}{15} \cdot|E(G)|$ edges do not satisfy the above condition. This bound is tight and the Petersen graph is the only connected graph for which the bound cannot be decreased. We obtain such a 4-edge-colouring by using a carefully chosen subset of edges of a perfect matching, and the analysis relies on a simple discharging procedure with essentially no reductions and very few rules.
\end{abstract}

Mathematics Subject Classifications: 05C15

*This work was partially supported by P.H.C. Proteus [37455VB]; ARRS [BI-FR-PROTEUS/17-18009, P1-0383, Project 1692]. 


\section{Introduction}

At the ninth British Combinatorial Conference in 1983, Fouquet and Jolivet [4] introduced strong edge-colourings of cubic graphs. This notion was further studied by Jaeger, who formulated a conjecture which is, arguably, one of the most challenging conjecture in graph theory. Proving Jaeger's conjecture to be true would have tremendous consequences, such as confirming the 5-Cycle double cover conjecture and the Berge-Fulkerson conjecture.

For any integer $k \geqslant 3$, consider a $k$-edge-colouring of a cubic graph $G=(V, E)$, that is, a mapping $f: E \rightarrow\{1, \ldots, k\}$ such that $f(e) \neq f\left(e^{\prime}\right)$ for every two edges $e$ and $e^{\prime}$ that share a vertex. For an edge $e \in E$, let $N(e)$ be the set of four edges adjacent to $e$. The edge $e$ is rich if $|f(N(e))|=4$, while it is poor if $|f(N(e))|=2$. The edge-colouring $f$ is normal if every edge is either rich or poor. The Petersen colouring conjecture reads as follows.

Conjecture 1 (The Petersen colouring conjecture - Jaeger, 1985). Every cubic bridgeless graph admits a normal 5-edge-colouring.

Now it is maybe a good time to explain the links with the ubiquitous Petersen graph $\mathscr{P}$. A Petersen colouring of a cubic graph $G=(V, E)$ is a mapping $g$ that associates to each edge of $G$ an edge of $\mathscr{P}$ such that if two edges $e$ and $e^{\prime}$ of $G$ share a vertex, then so do the edges $g(e)$ and $g\left(e^{\prime}\right)$ of $\mathscr{P}$. As observed by Jaeger [9], normal colourings and Petersen colourings of cubic graphs are in one-to-one correspondence.

Indeed, as is well known, the Petersen graph $\mathscr{P}$ can be seen as the Kneser graph with parameters 5 and 2, defined as follows: the vertices are in one-to-one correspondence with the 2-element subsets of $\{1, \ldots, 5\}$ and two vertices are adjacent if and only if the corresponding subset are disjoint. With this definition in mind, we can label every edge $u v$ of $\mathscr{P}$ by the unique integer $\ell(u v) \in\{1, \ldots, 5\}$ that does not belong to $X_{u} \cup X_{v}$, where $X_{w}$ is the 2-element subset of $\{1, \ldots, 5\}$ that corresponds to $w$, for every vertex $w$ of $\mathscr{P}$. Notice that if $g$ is a Petersen colouring of a cubic graph $G=(V, E)$, then $f: E \rightarrow\{1, \ldots, 5\}$ defined by $f(e):=\ell(g(e))$ is a normal colouring of $G$.

Conversely, assume that $f$ is a normal 5-edge-colouring of a cubic graph $G=(V, E)$. Keeping in mind the labelling $\ell$ of the edges of $\mathscr{P}$ given above, we define the mapping $g: E \rightarrow E(\mathscr{P})$ as follows. For each edge $e=u v \in E$, we define $g(e)$ to be the edge $e^{\prime}$ of $\mathscr{P}$ such that first $\ell\left(e^{\prime}\right)=f(e)$, and second $e^{\prime}$ is incident to the vertex $w \in V(\mathscr{P})$ such that the three colours assigned by $f$ to the edges of $G$ incident to $u$ are the elements of $X_{w} \cup\{f(e)\}$. A straightforward checking ensures that $g$ is a Petersen colouring of $G$. We just proved the following equivalence, which was first established by Jaeger [9].

Proposition 2. Let $G$ be a cubic graph. Then, $G$ admits a normal colouring if and only if $G$ admits a Petersen colouring.

Notice that a 3-edge-colouring of a (connected) cubic graph $G$ is precisely a normal colouring in which every edge is poor. Fouquet and Jolivet [4] coined the term strong colouring to define an edge-colouring in which every edge is rich. This corresponds precisely to an edge-colouring "at distance 2 " or, in other words, to a vertex-colouring of the 
square of the line graph of $G$. The unique normal colouring of the Petersen graph is a strong colouring.

Despite original approaches $[11,14,16]$, few progress has been made on the Petersen colouring conjecture: ways to infirm it remain elusive as possible counter-examples must be snarks, that is bridgeless cubic graphs that are not 3-edge-colourable (the ones we know are usually obtained from well-structured graph operations, for which the Petersen colouring conjecture can be verified $[2,6]$ ), and confirming the conjecture is expected to be a difficult task since as reported earlier this would confirm several difficult and most researched graph conjectures.

In view of the difficulty of the question, it is natural to ask for weaker versions of the conjecture. Because a strong colouring is normal, we know that every cubic graph admits a normal colouring using at most 10 colours: indeed Andersen [1] and, independently, Horák, He and Totter [8] established this statement (which confirms, for the particular case of graphs with maximum degree 3, a conjecture of Erdős and Nešetřil formulated in 1985 during a seminar in Prague). Further, it has been noted before [2] that Jaeger's 8-flow theorem provides, for any bridgeless cubic graph, a normal 7-edge-colouring. Actually, Mazzuoccolo and Mkrtchyan [12] even managed to extend this statement about 7-edgecolourings to all cubic graphs (not only bridgeless ones), which is best possible in the sense that there exist bridgeless cubic graphs that do not admit a normal 6 -edge-colouring). To the best of our knowledge, whether a normal 6-edge-colouring can be found for any such graph is still an open question. A line of study is then to find an edge-colouring that is normal on a large proportion of the graph, which we formalise in the next subsection. Before that, we end this part with a remark.

As mentioned earlier, a 3-edge-colourable graph $G$ always has a Petersen colouring: let $e_{1}, e_{2}$ and $e_{3}$ be the three edges incident to an arbitrary vertex $v$ of the Petersen graph. Label $e_{i}$ by $\ell\left(e_{i}\right):=i$ for each $i \in\{1,2,3\}$. If $c$ is a 3-edge-colouring of $G$, then defining $f: E(G) \rightarrow E(\mathscr{P})$ by $f\left(e^{\prime}\right):=e_{c\left(e^{\prime}\right)}$ yields a Petersen colouring of $G$. This Petersen colouring is, in some sense, trivial: it only uses the incidences at a single vertex of the Petersen graph. This is equivalent to saying that the original graph admits a 3edge-colouring. As first observed by Steffen (see [7, Proposition 2]), Petersen colourings of bridgeless cubic graphs are either "trivial" (and hence the graph admits a 3-edgecolouring) or surjective.

\subsection{The rich, the poor and the medium.}

Given an edge-colouring of a cubic graph $G$, define an edge $e$ to be medium if it is neither rich nor poor. Since the Petersen colouring conjecture states that every bridgeless cubic graphs admits a 5-edge-colouring such that no edge is medium, it seems interesting to investigate the minimum number of medium edges in edge-colourings of bridgeless cubic graphs. As observed by Bílková [2, p. 9], Petersen's perfect matching theorem combined with Vizing's edge-colouring theorem (and some further analysis if the graph has cycles of length less than 5) directly yield for every bridgeless cubic graph a 5-edgecolouring such that at least one third of the edges are rich or poor. This lower bound was improved [2, Theorem 3.2] to two thirds of the edges for cubic graphs having a 2-factor 
consisting of two cycles of the same length - the class of "generalised prisms" — and to roughly half the edges in graphs with no short cycles [2, Theorem 3.6].

Ways how poor, rich and medium edges combine in edge-colourings looks intriguing. We already pointed out that an edge-colouring with poor edges only is actually a 3-edgecolouring. Oppositely, an edge-colouring with rich edges only is a strong colouring. (As reported earlier, every normal 5-edge-colouring of the Petersen graph actually contains no poor edge.)

We consider 4-edge-colourings - in this case the number of colours assigned to the edges incident to any fixed edge is either 2 or 3 - and prove the following result.

Theorem 3. Every connected cubic bridgeless graph $G$ admits a 4-edge-colouring such that at most $\frac{8}{15} \cdot|E(G)|$ edges are neither rich nor poor. Furthermore, no 4-edge-colouring of $G$ yields less medium edges if and only if $G$ is the Petersen graph.

This bound indeed cannot be improved in general: the Petersen graph has 15 edges and each of its 4-edge-colourings yields at least 8 medium edges. This can be checked directly by case analysis (for example by discriminating edge-colourings according to the size of a smallest colour class), but it is rather tedious. We also verified it using an exhaustive search by computer.

Since an $n$-vertex cubic graph has $\frac{3 n}{2}$ edges, Theorem 3 ensures that every bridgeless cubic graph $G$ admits a 4 -edge-colouring containing at most $\frac{4}{5} \cdot|V(G)|$ medium edges. We use this formulation of the bound in terms of the number of vertices in the forthcoming proof of Theorem 3 .

\section{Proof of Theorem 3}

We demonstrate the upper bound: every bridgeless cubic graph $G$ admits a 4-edgecolouring such that at most $\frac{4}{5} \cdot|V(G)|$ edges are medium. While developing the proof, we shall see that the only case where the bound must be attained is if $G$ is the Petersen graph. We proceed by induction on the number of vertices of $G$. The induction yields the conclusion in a standard way if $G$ has a triangle, and we thus first deal with this case. To make the argument smoother, we actually prove the result for (loopless) bridgeless cubic multi-graphs. For instance any 4-edge-colouring of a triple edge between two vertices yields three poor edges. Similarly, if a cubic graph $G$ contains two vertices with exactly two parallel edges between them, then in any 4-edge-colouring of $G$ either both edges are poor or both edges are medium.

As reported earlier, every 3-edge-colouring of a cubic graph contains only poor edges, and hence the statement of Theorem 3 is correct if $G$ admits a 3-edge-colouring, and hence in particular if $|V(G)|=2$. We hence consider a connected bridgeless cubic multigraph $G$ that admits no 3-edge-colouring, and we set $n:=|V(G)|$ (so $n \geqslant 10$ ). Our first two arguments are standard and well known to people used to graph colouring but they are included for completeness.

We use induction to prove the statement if $G$ contains a multi-edge. Indeed, suppose that $e_{1}$ and $e_{2}$ are two different edges with end-vertices $v_{1}$ and $v_{2}$. For each $i \in\{1,2\}$, let $u_{i}$ 


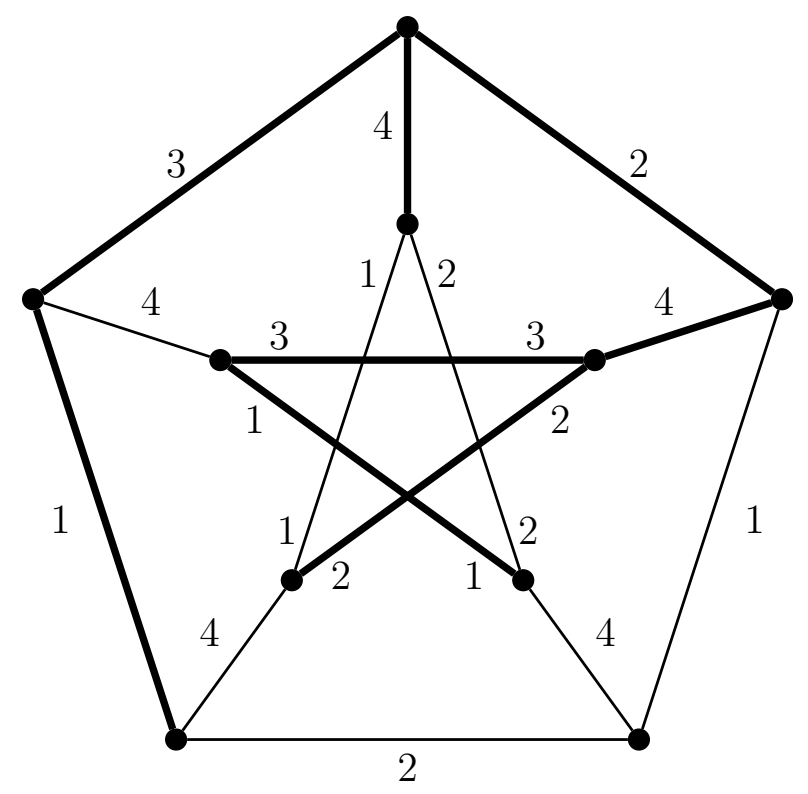

Figure 1: A 4-edge-colouring of the Petersen graph with exactly 8 medium edges, drawn thicker. The colouring is obtained by applying the construction explained in the proof of Theorem 3. (The colours of the inner edges are written near each of their end-vertices to improve readability and ease the checking.)

be the neighbour of $v_{i}$ different from $v_{3-i}$. Since $n>2$ and because $G$ is bridgeless, $u_{1} \neq u_{2}$. Let $G^{\prime}$ be the bridgeless cubic multi-graph obtained from $G-\left\{v_{1}, v_{2}\right\}$ by adding a new edge $e^{\prime}$ between $u_{1}$ and $u_{2}$. The induction hypothesis ensures that $G^{\prime}$ admits a 4-edgecolouring $c^{\prime}$ yielding at most $\frac{4}{5} \cdot(n-2)$ medium edges. It is straightforward to derive from $c^{\prime}$ a 4-edge-colouring $c$ of $G$ with no more medium edges, which thus prove the statement (including "the furthermore part") if $G$ contains a multi-edge. One obtains a 4-edge-colouring $c$ of $G$ by setting $c(e)=c^{\prime}(e)$ if $e \in E(G) \cap E\left(G^{\prime}\right)$ and $c\left(v_{i} u_{i}\right):=c^{\prime}\left(e^{\prime}\right)$ for each $i \in\{1,2\}$, and letting $c\left(e_{1}\right)$ and $c\left(e_{2}\right)$ be the two colours of the two edges of $G^{\prime}$ incident to $u_{1}$ that are different from $e^{\prime}$. The obtained 4-edge-colouring $c$ of $G$ yields at most $\frac{4}{5} \cdot(n-2)$ medium edges, which is less than $\frac{4}{5} \cdot n$. Consequently, we may assume that $G$ is simple.

We now use induction to prove the statement if $G$ contains a triangle $v_{0} v_{1} v_{2}$. Indeed, we then define $G^{\prime}$ to be the multi-graph obtained from $G$ by contracting $v_{0}, v_{1}$ and $v_{2}$ into a single vertex $x$. It follows that $G^{\prime}$ is a bridgeless and cubic multi-graph with $n-2$ vertices, and therefore the induction hypothesis yields that $G^{\prime}$ admits a 4-edge-colouring $c^{\prime}$ yielding at most $\frac{4}{5} \cdot(n-2)$ medium edges. This colouring $c^{\prime}$ can be extended into a 4-edgecolouring $c$ of $G$ by setting $c(e):=c^{\prime}(e)$ if $e \in E(G) \cap E\left(G^{\prime}\right)$ and $c\left(v_{i} v_{i+1}\right):=c^{\prime}\left(v_{i+2} u_{i+2}\right)$ where $i \in\{0,1,2\}$ is considered modulo 3 and $u_{i}$ is the neighbour of $v_{i}$ not in $\left\{v_{i+1}, v_{i+2}\right\}$. The obtained 4-edge-colouring $c$ yields no more medium edges in $G$ than $c^{\prime}$ does in $G^{\prime}$. Consequently, we may assume that $G$ is a simple bridgeless cubic graph with no triangle. 
It remains to deal with the case where $G$ is a bridgeless cubic graph with no triangles. Let $F$ a 2-factor of $G$ and $M$ the perfect matching such that $F=G-M$. We choose a 2-factor $F$ containing the smallest possible number of cycles. Because $G$ admits no 3-edge-colouring, we know that $F$ contains at least two odd cycles. In particular, there exists an edge in $M$ that is not a chord of a cycle in $F$. Note also that cycles of $G$ with length (at most) 5 have no chord, as $G$ has no triangle.

If $v \in V(G)$, we define $C_{v}$ to be the cycle in $F$ to which $v$ belongs and $v^{\prime}$ to be the unique neighbour of $v$ in $G$ such that $v v^{\prime} \in M$. For every cycle $C \in F$, there is a cyclic ordering $\varphi_{C}$ of the vertices in $V(C)$, which we extend to the edges in $M$ that are incident to a vertex in $C$ : two edges $e$ and $e^{\prime}$ in $M$ that have each exactly one end-vertex on a given cycle $C \in F$ are consecutive if their end-vertices in $C$ are consecutive with respect to $\varphi_{C}$. (Notice that chords are purposely excluded from this definition.)

An edge-selection is a subset $S$ of $M$ with the following properties:

1. every edge in $S$ is incident to two different odd cycles in $F$ (in particular, no edge in $S$ is a chord of a cycle in $F$ );

2. every cycle in $F$ is incident to at most two edges in $S$; and

3. if a cycle $C \in F$ is incident to two edges in $S$, then these two edges are consecutive.

Notice that the empty set is always an edge-selection (it might actually be the only one, for instance if no edge in $M$ links two different odd cycles in $F$ ). Given an edge-selection $S$ and a cycle $C \in F$, the degree $\operatorname{deg}_{S}(C)$ of $C$ in $S$ is the number of edges incident to $C$ that belong to $S$, and hence $\operatorname{deg}_{S}(C) \in\{0,1,2\}$. If in addition $C^{\prime} \in F$, then $C$ and $C^{\prime}$ are $S$-adjacent if $S$ contains an edge incident to both $C$ and $C^{\prime}$. An $S$-component of $G$ is an inclusion-wise maximal subset $K$ of $F$ such that for every two distinct cycles $C$ and $C^{\prime}$ in $K$, there exists a sequence $\left(C_{i}\right)_{0 \leqslant i \leqslant t}$ of cycles in $K$ such that $C_{0}=C, C_{t}=C^{\prime}$ and for every $i \in\{0, \ldots, t-1\}$, the cycles $C_{i}$ and $C_{i+1}$ are $S$-adjacent. The edges in $S$ joining two cycles in $K$ are said to be associated with $K$. We need a last definition. If $K$ is an $S$-component, then we let $G_{K}$ be the multi-graph with vertex set $K$ and $x$ edges between $C$ and $C^{\prime}$ where $x \in\{0,1,2\}$ is the number of edges in $S$ that are incident to both $C$ and $C^{\prime}$ in $G$. It follows from the definitions that $G_{K}$ is either a single vertex, or a path, or a cycle, or two vertices joined by two parallel edges. However, because $F$ minimises the number of cycles in a 2 -factor of $G$, the last two cases cannot occur. Indeed, if $G_{K}$ is a cycle $u_{0}, \ldots, u_{k-1}$ with $k \geqslant 2$, then $F$ contains $k$ different cycles $v_{0}^{i}, \ldots, v_{t_{i}-1}^{i}$ for $i \in\{0, \ldots, k-1\}$, where the vertex names can be chosen such that

$$
v_{1}^{0} v_{0}^{1} v_{1}^{1} v_{0}^{2} v_{1}^{2} \ldots v_{0}^{k-1} v_{1}^{k-1} v_{0}^{0}
$$

is an even cycle in $G$ with exactly half its edges in $M$. Therefore, inverting the edges in and out of $M$ along $C$ yields a 2 -factor of $G$ with fewer cycles than $F$, a contradiction. (See Figure 2 for an illustration.)

Now, among all edge-selections of maximum order, we choose one such that the number of $S$-degree-2 cycles is as large as possible. We construct a 4 -edge-colouring of $G$ with the following properties: 


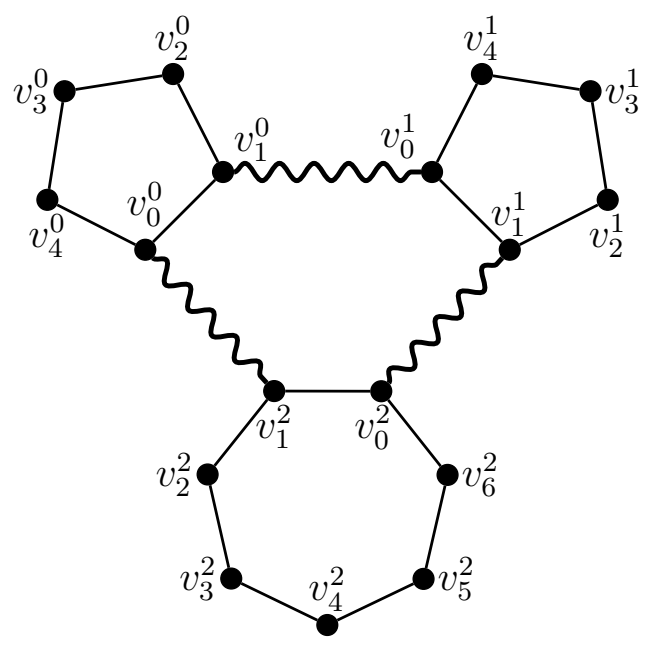

Figure 2: Illustration that $G_{K}$ cannot be a cycle, with $k=3$. The waved edges are those in $S$.

- every edge in $M$ is coloured 4 and no other edge is coloured 4;

- an edge is coloured 3 only if it belongs to an odd cycle in $F$;

- every odd cycle in $F$ has exactly one edge coloured 3;

- every edge in $S$ is adjacent to two edges coloured 3; and

- no edge in $S$ is medium.

To see why such a 4-edge-colouring exists, start by colouring the edges of $G$ that belong to $M$ with 4 . Next colour the edges of every even cycle in $F$ using $\{1,2\}$. By (2) and (3) every odd cycle $C \in F$ has an edge that is incident to all the edges in $S$ incident to $C$ : colour this edge with 3 . The remaining uncoloured edges span a vertex-disjoint collection of paths, and we colour them using $\{1,2\}$, independently for each $S$-component $K$. Concretely, choose an ordering of the paths in the $S$-component and colour arbitrarily the first one. When considering the $i^{\text {th }}$ path in the component, with $i \geqslant 2$, we choose its colouring so that the edge in $S$ between between the $i^{\text {th }}$ and the $(i-1)^{\text {th }}$ path is not medium. Therefore, as $G_{K}$ is a path we created no medium edge in $S$ during the procedure (because paths and even cycles are 2-colourable).

Our goal is to prove that the obtained 4-edge-colouring of $G$ contains at most $4 n / 5$ medium edges, where $n$ is the number of vertices of $G$. We use a discharging argument to count the medium edges: we start by assigning a charge of 1 to each medium edge, and thus throughout all the process the total charge in the graph $G$ is precisely the number of medium edges.

We shall define a number of discharging rules: in the first ones, medium edges send charge to cycles in $F$ to which they are incident. Later, some cycles in $F$ will send some 


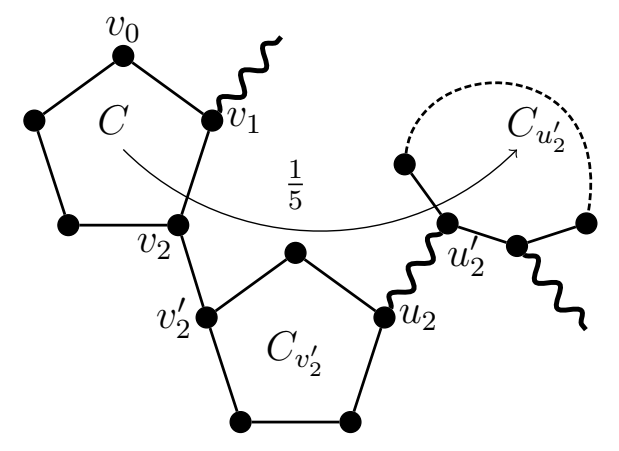

Figure 3: Illustration of the discharging rule (R4): $C$ and $C_{v_{2}^{\prime}}$ both are cycles of length 5 and $S$-degree 1 while $C_{u_{2}^{\prime}}$ has $S$-degree 2; waved edges are those in $S$ and one could have $C_{v_{1}^{\prime}}=C_{u_{2}^{\prime}}$, that is, the two waved edges with only one end-vertex drawn could be the same edge.

charge to other cycles in $F$. We apply the rules in order and analyse the global state of the charge in the graph after one or more rules have been applied. At the end, we prove that for each $S$-component $K$ the sum of the charges of the cycles in $K$ is at most $\frac{4}{5}$ times the number of vertices belonging to cycles in $K$, which implies the sought upper bound on the number of medium edges.

Fact 4 . A cycle in $F$ contains no medium edge if it is even and 3 medium edges if it is odd.

Proof. Indeed, if the edge $e$ belongs to an even cycle, then its colour $c$ belongs to $\{1,2\}$, and each of its end-vertices is incident to an edge coloured 4, which belongs to $M$, and an edge coloured $3-c$, which belongs to the even cycle. So $e$ is poor. Let $C=v_{0} \ldots v_{2 k}$ be an odd cycle in $F$ with $v_{0} v_{1}$ being its only edge coloured 3. For each $i \in\{2, \ldots, 2 k-1\}$, let $c_{i} \in\{1,2\}$ be the colour of the edge $v_{i} v_{i+1}$. Then the edge $v_{i} v_{i+1}$ is incident to two edges coloured 4 and two edges coloured $3-c_{i}$, and hence $v_{i} v_{i+1}$ is poor. Consequently, the only medium edges on $C$ are $v_{0} v_{1}, v_{1} v_{2}$ and $v_{2 k} v_{0}$.

Our first rule reads as follows.

(R0) Every medium edge that belongs to a cycle $C$ in $F$ sends 1 to $C$.

After applying rule (R0), an edge has charge 0 except if it is a medium edge that belongs to $M$, in which case it has charge 1 . In addition, a cycle in $F$ has charge 0 if it is even and charge 3 if it is odd. For our next rule, notice that if $e$ is a medium edge that belongs to $M$, then $e$ is adjacent to an edge coloured 3, which must belong to an odd cycle in $F$.

(R1) Let $e$ be a medium edge that belongs to $M$, and let $C$ and $C^{\prime}$ be the two cycles in $F$ to which $e$ is incident, such that $C$ is odd and the edge coloured 3 on $C$ is adjacent to $e$. 
If $C^{\prime}$ is even, then $e$ sends $1 / 2$ to $C^{\prime}$ and $1 / 2$ to $C$. If $C^{\prime}$ is odd and its edge coloured 3 is adjacent to $e$, then $e$ sends $1 / 2$ to each of $C$ and $C^{\prime}$. If $C^{\prime}$ is odd and its edge coloured 3 is not adjacent to $e$, then $e$ sends 1 to $C$.

After applying rule (R1), every edge has charge 0 . If $C \in F$ is an even cycle, then its charge is at most $1 / 2 \cdot|V(C)|$, which is less than $4|V(C)| / 5$. In addition, if $C \in F$ is an odd cycle, then one of the following occurs:

- $C$ has $S$-degree 0 and charge at most 5;

- $C$ has $S$-degree 1 and charge at most 4; or

- $C$ has $S$-degree 2 and charge at most 3 .

Indeed, edges in $S$ are not medium by the construction of the 4-edge-colouring. It follows that the charge of $C$ is at most $\frac{4}{5} \cdot|V(C)|$ unless $C$ has length 5 and $S$-degree 0 .

The next step is to apply the three following rules, the third one being illustrated in Figure 3. For these rules, we sometimes want to emphasise not only the cycles involved, but also a particular vertex of the configuration that belongs to the cycle sending, or receiving, charge: to identify such a vertex $v$, we say that the charge is sent, or received, through $v$.

(R2) If $C$ is a cycle of length 5 in $F$ with $S$-degree 0 , then $C$ sends $1 / 5$ to $C_{v^{\prime}}$ for each $v \in V(C)$.

(R3) Let $C=v_{0} \ldots v_{4}$ be a cycle of length 5 in $F$ of $S$-degree 1 , with $v_{1} v_{1}^{\prime}$ being the unique edge in $S$ incident to $C$. For each $i \in\{0,2\}$, if $C_{v_{i}^{\prime}}$ is not a cycle of length 5 with $S$-degree 1 , then $C$ sends $1 / 5$ to $C_{v_{i}^{\prime}}$. The charge is sent through $v_{i}$ and received through $v_{i}^{\prime}$.

(R4) Let $C=v_{0} \ldots v_{4}$ be a cycle of length 5 in $F$ of $S$-degree 1 , with $v_{1} v_{1}^{\prime}$ being the unique edge in $S$ incident to $C$. Let $i \in\{0,2\}$. Suppose that $C_{v_{i}^{\prime}}$ is a cycle of length 5 of $S$-degree 1 , written $v_{i}^{\prime} u_{1} u_{2} u_{3} u_{4}$ such that $u_{2}$ is its unique vertex incident to an edge in $S$. If $C_{u_{2}^{\prime}}$ is a cycle of $S$-degree 2 then $C$ sends $1 / 5$ to $C_{u_{2}^{\prime}}$. The charge is sent through $v_{i}$ and received through $u_{2}^{\prime}$.

We now check that after applying (R2)-(R4), for every cycle $C$ in $F$, the charge of $C$ is at most $4 / 5$ times the number of vertices belonging to $C$ with equality only if $C$ has length 5 .

If $C$ is an even cycle, then it has charge at most $(1 / 2+1 / 5) \cdot|V(C)|<\frac{4}{5} \cdot|V(C)|$.

If $C$ is a cycle with $S$-degree 0 and length $2 k+1$, where $k \geqslant 2$, then $C$ does not receive any charge. Indeed, there cannot be an edge $e$ in $M$ incident to $C$ and a cycle of length 5 and $S$-degree 0, as $S \cup\{e\}$ would then contradict the maximality of $S$. This shows that $C$ does not receive charge by (R2). Moreover, if $C$ would receive charge by (R3) then the definition of (R3) would imply the existence of a cycle $C^{\prime}=v_{0} v_{1} v_{2} v_{3} v_{4}$ in $F$ of length 5 and $S$-degree one such that $v_{1} v_{1}^{\prime} \in S$ and $C=C_{v_{0}^{\prime}}\left(\right.$ or $\left.C=C_{v_{2}^{\prime}}\right)$. Consequently, $S \cup\left\{v_{0} v_{0}^{\prime}\right\}$ would contradict the maximality of $S$. Therefore, the final charge of $C$ is at most 5 
if $k \geqslant 3$, which is less than $4 \cdot(2 k+1) / 5$. If $k=2$ then by rule (R2) the final charge of $C$ is at most $5-5 \cdot \frac{1}{5}=4=4 \cdot(2 k+1) / 5$.

Let $C$ be a cycle with $S$-degree 1 . Note that $C$ can receive charge only because of (R2) and (R3). Moreover, $C$ does not receive charge through its vertex incident to an edge in $S$. If $C$ has length $2 k+1$ with $k \geqslant 3$, then before applying (R2)-(R4) the charge of $C$ was at most 4 since the edge in $S$ incident to $C$ is not medium, and hence its final charge is at most $4+2 k / 5$, which is less than $4 \cdot(2 k+1) / 5$ since $k \geqslant 3$.

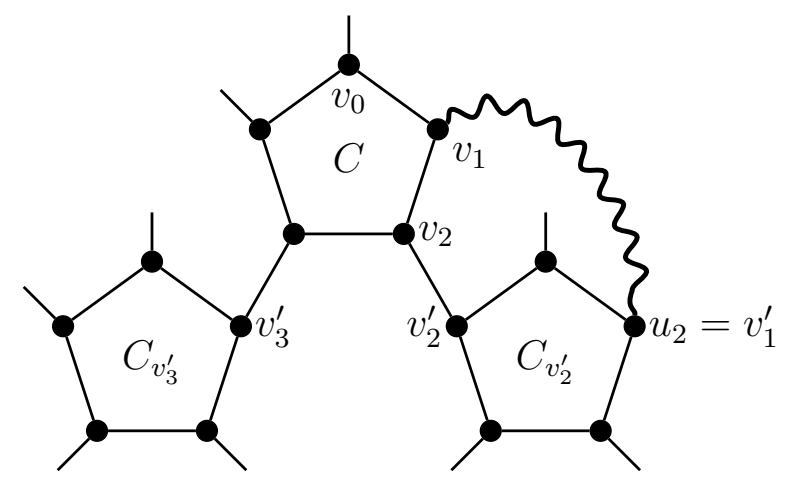

Figure 4: If $C$ and $C_{v_{2}^{\prime}}$ are $S$-adjacent cycles of length 5 with $S$-degree 1 , then $C_{v_{3}^{\prime}}$ cannot be a cycle of length 5 with $S$-degree 0 , for otherwise $\left(S \backslash\left\{v_{1} v_{1}^{\prime}\right\}\right) \cup\left\{v_{2} v_{2}^{\prime}, v_{3} v_{3}^{\prime}\right\}$ would contradict the maximality of $S$. Consequently, $C_{v_{3}^{\prime}}$, does not send charge to $C$ by (R2).

If $C$ has length 5 , then let us write $C=v_{0} \ldots v_{4}$ with $v_{1} v_{1}^{\prime} \in S$. Recall that $v_{1} v_{1}^{\prime}$ cannot be medium because no edge in $S$ is medium. Furthermore, $C$ can receive charge only by (R2). Observe that none of $C_{v_{0}^{\prime}}$ and $C_{v_{2}^{\prime}}$ is an odd cycle with $S$-degree 0 for otherwise adding the edge $v_{0} v_{0}^{\prime}$ or $v_{2} v_{2}^{\prime}$ to $S$ would contradict its choice. Therefore $C$ can receive charge only through $v_{3}$ or $v_{4}$, for a total of at most $2 / 5$. It follows that if $C$ sends $1 / 5$ through each of $v_{0}$ and $v_{2}$, due to rules (R3) and (R4), then its final charge surely is at most $\frac{4}{5} \cdot|V(G)|$. Let us identify precisely when $C$ sends charge through $v_{2}$, the case for $v_{0}$ being identical. If $C_{v_{2}^{\prime}}$ is not a cycle of length 5 with $S$-degree 1 , then (R3) applies. So assume that $C_{v_{2}^{\prime}}$ is a cycle of length 5 with $S$-degree 1 , written $v_{2}^{\prime} u_{1} u_{2} u_{3} u_{4}$. None of $u_{1}$ and $u_{4}$ is incident to an edge in $S$, for otherwise adding the edge $v_{2} v_{2}^{\prime}$ to $S$ would contradict its maximality. So we can assume without loss of generality that $u_{2} u_{2}^{\prime} \in$ $S$. Now we observe that if $C_{u_{2}^{\prime}} \neq C$, then $C_{u_{2}^{\prime}}$ cannot be a cycle of $S$-degree 1 , for otherwise $\left(S \backslash\left\{u_{2} u_{2}^{\prime}\right\}\right) \cup\left\{v_{2} v_{2}^{\prime}\right\}$ would contradict the fact that $S$, among all edge-selections of maximum order, creates the maximum number of cycles with $S$-degree 2 . Therefore in this case $C_{u_{2}^{\prime}}$ is a cycle of $S$-degree 2 and hence $C$ sends $1 / 5$ to $C_{u_{2}^{\prime}}$ by (R4). It follows that the only case where $C$ does not send charge through $v_{2}$ is when $C_{u_{2}^{\prime}}=C$ and hence $v_{1}^{\prime}=u_{2}$ (or $v_{1}^{\prime}=u_{3}$ ). In this situation, we argue that $C$ cannot receive charge through $v_{3}$. Indeed $C$ can receive charge through $v_{3}$ only by (R2), which applies if and only if $C_{v_{3}^{\prime}}$ is a cycle of length 5 and $S$-degree 0, as illustrated in Figure 4. In this case, $\left(S \backslash\left\{v_{1} v_{1}^{\prime}\right\}\right) \cup\left\{v_{2} v_{2}^{\prime}, v_{3} v_{3}^{\prime}\right\}$ would contradict the maximality of $S$. Consequently, we proved that either $C$ sends $1 / 5$ through $v_{2}$ or $C$ receives nothing through $v_{3}$. By symmetry of the roles played by $v_{2}$ 
and $v_{0}$, either $C$ sends $1 / 5$ through $v_{0}$ or $C$ receives nothing through $v_{4}$. Since $C$ can receive charge only through $v_{3}$ and $v_{4}$, we therefore conclude that the final charge of $C$ is not greater than its charge before applying (R2)-(R4), that is 4 .

It remains to deal with cycles with $S$-degree 2 . Let us write $C=v_{0} \ldots v_{2 k}$, where $k \geqslant 2$, with $v_{1} v_{1}^{\prime} \in S$ and $v_{2} v_{2}^{\prime} \in S$. In particular, none of $v_{1} v_{1}^{\prime}$ and $v_{2} v_{2}^{\prime}$ is medium, and thus the charge of $C$ after applying (R0) and (R1) is 3. We show that after applying the other rules, the charge of $C$ is at most $\frac{4}{5} \cdot(2 k+1)$, with equality only if $k=2$. Observe that, for each $i \in\{1,2\}$, the cycle $C$ can receive some charge through $v_{i}$ only if $C_{v_{i}^{\prime}}$ is a cycle of length 5 and $S$-degree 1 . Further, according to (R4), the cycle $C$ can receive at most $2 / 5$ through $v_{i}$, because only the two vertices at distance two from $v_{i}^{\prime}$ on $C_{v_{i}^{\prime}}$ can be involved in an application of (R4). As a result, it is enough to prove that if $C$ receives $2 / 5$ through $v_{i}$, then $C$ does not receive any charge through $v_{6-3 i}$. More explicitly, if $C$ receives 2/5 through $v_{1}$ then $C$ receives nothing through $v_{3}$; and if $C$ receives $2 / 5$ through $v_{2}$ then $C$ receives nothing through $v_{0}$. In total, the final charge of $C$ would then be at most $3+(2 k+1) / 5$, which is at most $\frac{4}{5} \cdot(2 k+1)$ since $k \geqslant 2$, with equality if and only if $k=2$.

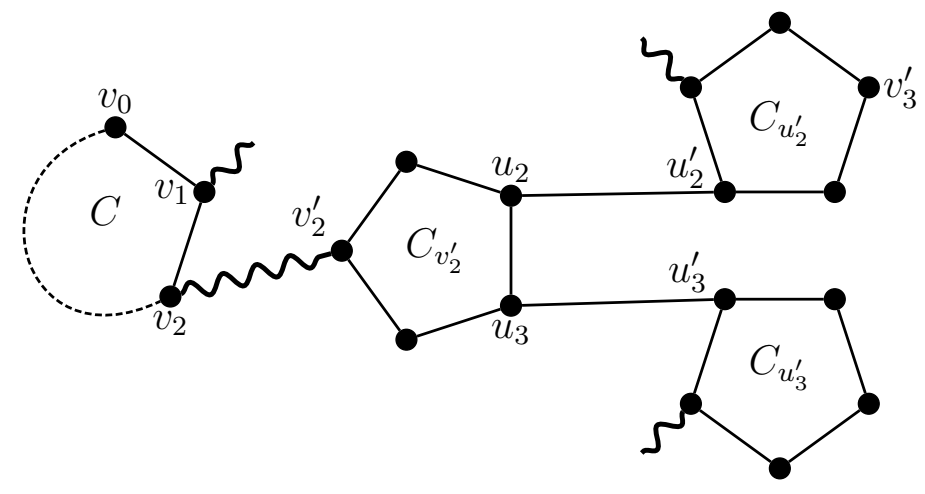

Figure 5: If the cycle $C$ receives $2 / 5$ through $v_{2}$ by (R4) and $C_{u_{2}^{\prime}} \neq C_{u_{3}^{\prime}}$ then $\left(S \backslash\left\{v_{2} v_{2}^{\prime}\right\}\right) \cup$ $\left\{u_{2} u_{2}^{\prime}, u_{3} u_{3}^{\prime}\right\}$ contradicts the maximality of the edge-selection $S$.

Let us establish the assertion above: assume without loss of generality that $C$ receives $2 / 5$ through $v_{2}$ because of (R4). Writing $C_{v_{2}^{\prime}}=v_{2}^{\prime} u_{1} u_{2} u_{3} u_{4}$, we deduce that each of $C_{u_{2}^{\prime}}$ and $C_{u_{3}^{\prime}}$ is a cycle of length 5 and $S$-degree 1 . In addition, by the definition of (R4) for each $i \in\{2,3\}$ the vertex of $C_{u_{i}^{\prime}}$ incident to an edge in $S$ is a neighbour of $u_{i}^{\prime}$ on $C_{u_{i}^{\prime}}$. Suppose first that $C_{u_{2}^{\prime}} \neq C_{u_{3}^{\prime}}$, as illustrated in Figure 5. Then $\left(S \backslash\left\{v_{2} v_{2}^{\prime}\right\}\right) \cup\left\{u_{2} u_{2}^{\prime}, u_{3} u_{3}^{\prime}\right\}$ contradicts the maximality of $S$. If, on the contrary, $C_{u_{2}^{\prime}}=C_{u_{3}^{\prime}}$, then without loss of generality we may write $C_{u_{2}^{\prime}}=w_{0} u_{2}^{\prime} w_{1} w_{2} u_{3}^{\prime}$ with $w_{0} w_{0}^{\prime} \in S$, as illustrated in Figure 6 . Now, if $C$ receives charge through $v_{0}$ then $C_{v_{0}^{\prime}}$ is a cycle of length 5 and $S$-degree 0 or 1 . In the latter case, we notice that the vertex of $C_{v_{0}^{\prime}}$ incident to an edge in $S$ is consecutive to $v_{0}^{\prime}$ on $C_{v_{0}^{\prime}}$ and, consequently in both cases $C_{v_{0}^{\prime}} \neq C_{u_{2}^{\prime}}$. From this and the fact that $v_{0}^{\prime} \notin\left\{u_{2}^{\prime}, u_{3}^{\prime}\right\}$, we deduce that in any case $\left(S \backslash\left\{v_{2} v_{2}^{\prime}\right\}\right) \cup\left\{v_{0} v_{0}^{\prime}, u_{2} u_{2}^{\prime}\right\}$ contradicts the choice of $S$. 


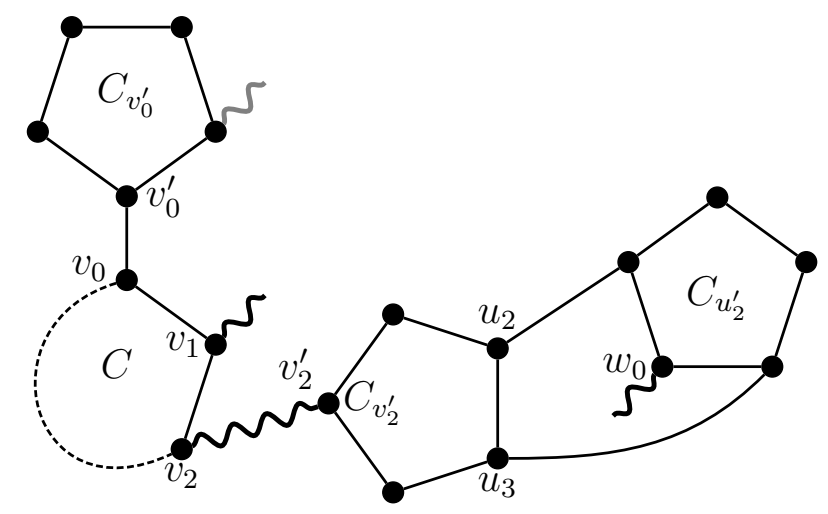

Figure 6: If the cycle $C$ receives $2 / 5$ through $v_{2}$ by $(\mathrm{R} 4)$ and $C_{u_{2}^{\prime}}=C_{u_{3}^{\prime}}$, then $C$ cannot receive $1 / 5$ through $v_{0}$ by (R2) or (R3), for otherwise $\left(S \backslash\left\{v_{2} v_{2}^{\prime}\right\}\right) \cup\left\{v_{0} v_{0}^{\prime}, u_{2} u_{2}^{\prime}\right\}$ would contradict the maximality of the edge-selection $S$. (The gray waved edge belongs to $S$ only if $C_{v_{0}^{\prime}}$ has $S$-degree 1.)

Looking at the above inequalities, we observe that as soon as $F$ contains a cycle $C$ of length different from 5 , then the number of medium edges is less than $\frac{4}{5} \cdot|V(G)|$. Consequently, if the number of medium edges is $\frac{4}{5} \cdot|V(G)|$, then we can assume that every 2-factor of $G$ that minimises the number of cycles contains only cycles of length 5 . This implies that no 2-factor of $G$ contains a cycle of length different from 5 , since $G$ is cubic and triangle-free. Indeed, let $F$ be a 2-factor minimising the number of cycles, and suppose that $F^{\prime}$ is a 2-factor of $G$ that contains a cycle of length different from 5 . Necessarily, $F^{\prime}$ contains more cycles than $F$. Since $G$ is simple and has no triangle, and $F$ contains only cycles of length 5 , we deduce that $F^{\prime}$ must contain a 4-cycle $C^{\prime}$. Further, there must exit two cycles $C_{0}=v_{0} \ldots v_{4}$ and $C_{1}=u_{0} \ldots u_{4}$ in $F$ such that $C^{\prime}$ is $v_{0} u_{0} u_{4} v_{1}$, see Figure 7 . Therefore removing $C_{0}$ and $C_{1}$ from $F$ and adding $v_{0} u_{0} \ldots u_{4} v_{1} \ldots v_{4}$ yields a 2-factor of $G$ with fewer cycles than $F$, which is a contradiction. Therefore, if the 4-edge-colouring produced contains exactly $\frac{4}{5} \cdot|V(G)|$ then every 2 -factor of $G$ contains only cycles of length 5. As it turns out, it has been proved [3] that every connected bridgeless cubic graph different from the Petersen graph admits a 2-factor containing a cycle of length different from $5^{1}$. This concludes the proof of Theorem 3 .

\section{Further work}

We point out that, using more involved discharging rules and a lengthier analysis, one can show that there exists a positive $\varepsilon$ (which we did not try to optimise) such that for every connected bridgeless cubic graph $G$ different from the Petersen graph, there exists a 4-edge-colouring yielding at most $(4 / 5-\varepsilon)|V(G)|$ medium edges.

It seems stimulating to try and obtain upper bounds for the least possible number of

\footnotetext{
${ }^{1}$ See also [10] for a different and short argument.
} 


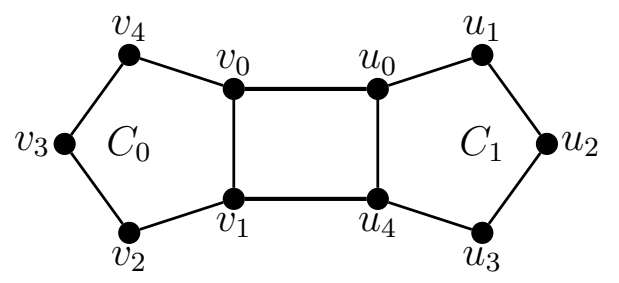

Figure 7: A 4-cycle $C^{\prime}$ of $G$ must have exactly two edges in $F$ and two outside of $F$ (those are in bold).

medium edges in a $k$-edge-colouring of a bridgeless cubic graph. As we saw, this number is 0 if $k \geqslant 7$ and at most $\frac{4}{5} \cdot|V(G)|$ if $k=4$. Since the Petersen colouring conjecture states that this number should be 0 when $k=5$, can one obtain at least a sub-linear (in the number of vertices) upper bound in this case? Mazzuoccolo and Mkrtchyan recently followed the same line of thoughts and studied this question allowing 6 colours: they proved [13] that one can find for every cubic bridgeless graph a 6-edge-colouring such that at most $\frac{2}{9} \cdot|E(G)|$ are medium. Even with 6 colours, it seems interesting to us to try and develop an argument to prove that a sublinear number of medium edges can be guaranteed.

\section{Acknowledgements}

We thank both referees for their careful reading, and one of them for pointing to us that choosing a 2-factor minimising the number of cycles would simplify one case of the analysis; but then restricting to such a 2-factor required an extra argument to show that the Petersen graph is the only graph for which the bound cannot be improved. On the other hand, we point out the 4-edge-colouring we construct actually works with any 2 -factor, as shown in the original version [15].

\section{References}

[1] L. D. Andersen, The strong chromatic index of a cubic graph is at most 10, Discrete Math. 108 (1992), no. 1-3, 231-252.

[2] H. Bílková, Variants of Petersen coloring for some graph classes, Charles University in Prague, 2015. Master Thesis.

[3] M. DeVos, V. V. Mkrtchyan, and S. S. Petrosyan, 5-cycles and the Petersen graph, arXiv:0801.3714, unpublished.

[4] J.-L. Fouquet and J.-L. Jolivet, Strong edge-colorings of graphs and applications to multi-k-gons, Ars Combin. 16 (1983), no. A, 141-150.

[5] T. Kaiser and J.-S. Sereni, unpublished. 
[6] J. Hägglund and E. Steffen, Petersen-colorings and some families of snarks, Ars Math. Contemp. 7 (2014), 161-173.

[7] A. Hakobyana and V. Mkrtchyan, $S_{12}$ and $P_{12}$-colorings of cubic graphs, Ars Math. Contemp. 17 (2019), no. 2, 431-445.

[8] P. Horák, Q. He, and W. T. Trotter, Induced matchings in cubic graphs, J. Graph Theory 17 (1993), no. 2, 151-160.

[9] F. Jaeger, On five-edge-colorings of cubic graphs and nowhere-zero flow problems, Ars Combin. 20 (1985), no. B, 229-244.

[10] D. Král'. Personnal communication to the second author; see https://lbgi.fr/ sereni/Lectures/AG_Fal109/ag09_3.pdf.

[11] E. Máčajová and M. Škoviera, Fano colourings of cubic graphs and the Fulkerson conjecture, Theoret. Comput. Sci. 349 (2005), no. 1, 112-120.

[12] G. Mazzuoccolo and V. Mkrtchyan, Normal edge-colorings of cubic graphs, J. Graph Theory, forthcoming, doi:10.1002/jgt.22507.

[13] _ Normal 6-edge-colorings of some bridgeless cubic graphs, Discrete Appl. Math., forthcoming, doi:10.1016/j.dam.2019.09.019.

[14] J. Nešetřil and R. Šámal, On tension continuous mappings, European J. Combin. 29 (2008), no. 4, 1025-1054.

[15] F. Pirot, J.-S. Sereni, and R. Škrekovski, Variations on the Petersen colouring conjecture, arXiv:1905.07913, unpublished.

[16] R. Šámal, New approach to Petersen coloring, Electr. Notes Discrete Math. 38 (2011), $755-760$. 